\title{
Cumulative Sum Analysis of the Learning Curve of Ptosis Surgery: External Levator Advancement versus Müller Muscle-conjunctival Resection
}

\author{
Mehmet Serhat Mangan ${ }^{1}$, Akin Cakir², Serhat Imamoglu ${ }^{1}$ \\ ${ }^{1}$ Division of Oculoplastic and Reconstructive Surgery, Sadik Eratik Eye Institute, Haydarpasa Numune Education and Research \\ Hospital, University of Health Sciences, Istanbul, Turkey \\ ${ }^{2}$ Department of Ophthalmology, Okmeydani Education and Research Hospital, University of Health Sciences, Istanbul, Turkey
}

Purpose: Even though it is stated that external levator advancement (ELA) has a much longer learning curve than Müller muscle-conjunctival resection (MMCR) in the treatment of aponeurotic ptosis, there is no study in the literature regarding the learning curves of these two surgical techniques. We aimed to objectively determine the length of the learning curves of ELA and MMCR using cumulative sum (CUSUM) analysis.

Methods: The first 30 unilateral ELA and the first 30 unilateral MMCR consecutively performed by a single surgeon were retrospectively reviewed. The CUSUM method was used to analyze the learning curves of ELA and MMCR based on operation times of consecutive surgeries and the clinical outcomes were compared.

Results: CUSUM analyses revealed that the operation time stabilized after around 11 ELA surgeries and 12 MMCR surgeries and $R^{2}$ value for ELA and MMCR were 0.93 and 0.91, respectively. There was no significant difference in these stratified analyses. Mean operation time was 45.7 minutes in the ELA group and 34.1 minutes in the MMCR group $(p=0.002)$. Total number of intraoperative complications was 37 in the ELA group and 16 in the MMCR group $(p=0.015)$. Symmetry success rate was significantly higher $(p<0.0001)$ and reoperation rate was significantly lower in the MMCR group $(p=0.045)$.

Conclusions: Even though ELA is more challenging than MMCR, comparable learning curves indicate that surgeons in training need to be encouraged to perform both techniques. The challenges and obstacles that the surgeons in training face in these two techniques need to be analyzed in detail.

Key Words: Blepharoptosis, Cumulative sum analysis, External levator advancement, Learning curve analysis, Müller muscleconjunctival resection

Received: April 15, 2021 Final revision: June 25, 2021

Accepted: July 15, 2021

Corresponding Author: Mehmet Serhat Mangan, MD. Division of Oculoplastic and Reconstructive Surgery, Sadik Eratik Eye Institute, Haydarpasa Numune Education and Research Hospital, University of Health Sciences, Tibbiye Cad. No:23, Istanbul 34668, Turkey. Tel: 90-530-1093800, Fax: 90-216-336-0565, E-mail: mehmetsmangan@yahoo.com
External levator advancement (ELA) and Müller muscle-conjunctival resection (MMCR) are the most common techniques used in the treatment of aponeurotic ptosis, yet the treatment of choice remains controversial [1-8]. Along with the severity of ptosis and response to phenylephrine test, surgeon's preference and experience may play a major role in the choice of surgical technique [1-9]. A question- 
naire of members of the American Society of Ophthalmic and Reconstructive Surgery about blepharoptosis surgery reported that $74 \%$ of surgeons performed posterior approach ptosis surgery [9]. This preference may be affected by the learning curve of surgeons in training. Classically, ELA is known to have a longer learning curve than MMCR. ELA having longer operation time and more unpredictable outcomes may indicate a more challenging operation, but these data does not suffice to analyze its learning curve.

Even though the parameters that determine the learning curve of a single surgical technique vary in different studies, rates of surgical success, intraoperative and postoperative complications, and reoperation are commonly used [10]. However, more objective analytic methods are required when comparing two different surgical techniques. Therefore, the cumulative sum (CUSUM) analysis has been developed to assess the learning curves of surgical procedures and is accepted in ophthalmology and many surgical fields [11-17]. CUSUM analysis, which is a statistical process control method, provides an objective and quantitative evaluation of surgical learning curves [11-21].

Recently, studies with large patient series that compare the clinical outcomes of ELA and MMCR have been published [4-7]. These studies reported the outcomes of both bilateral and unilateral ptosis surgeries and there is no study that reports the outcomes of solely unilateral ptosis surgery. Also, even though it is classically known that ELA has a longer learning curve that MMCR, to the best of our knowledge, there is no study that compares the learning curves of ELA and MMCR as well as their clinical outcomes.

Analyzing the learning curves of ELA and MMCR operations may yield a new perspective for the training of surgical residents and/or fellows. Therefore, we aimed to objectively determine the length of the learning curves of ELA and MMCR operations using CUSUM analysis.

\section{Materials and Methods}

\section{Study design}

This study was approved by the institutional review board of University of Health Sciences, Haydarpasa Numune Education and Research Hospital (HNEAH-KAEK 2020/205) and was conducted in accordance with the tenets of the Declaration of Helsinki. Written informed consent was obtained from all patients. Patients who underwent unilateral ptosis repairment (MMCR vs. ELA) between January 2014 and January 2018 by a single surgeon were retrospectively analyzed.

The inclusion criteria for the study were as follows: (1) diagnosis of aponeurotic ptosis with levator function of more than $10 \mathrm{~mm}$, (2) the first 30 unilateral MMCR and the first 30 unilateral ELA operations consecutively performed by a single surgeon, and (3) patients older than 25 years.

Patients meeting any of the following criteria were excluded: (1) patients undergoing additional surgery (blepharoplasty, brow lift, etc.) in the same session with unilateral ptosis surgery, (2) patients undergoing bilateral ptosis surgery, (3) patients undergoing frontalis suspension surgery, (4) previous surgery on the same eyelid, (5) congenital, traumatic, neurogenic or myogenic causes, (6) missing preoperative or postoperative records, (7) patients with a follow-up period shorter than 3 months, and (8) patients who had a systemic disorder increasing the tendency to bleed or those where it was not possible to stop the anticoagulant treatment preoperatively.

\section{Patient evaluation}

Unilateral ptosis was defined as margin-reflex distance (MRD1) being $>1.5 \mathrm{~mm}$ lower than the contralateral eyelid $[22,23]$. Prior to ptosis repair, levator function and MRD1 were measured in both eyes of all patients. At least three measurements were performed with the same ruler in all cases at the clinic on the same day.

MMCR was only considered if there was an adequate response (defined as eyelid height difference of $\geq 1.5 \mathrm{~mm}$, preand post-phenylephrine) and the eyelid crease symmetry was achieved after administration of two drops of $2.5 \%$ phenylephrine with 5-minute intervals into the upper conjunctival fornix of the more ptotic eyelid. ELA was considered in the following cases: poor response (defined as eyelid height difference of $<1.5 \mathrm{~mm}$, pre- and post-phenylephrine) or eyelid height and/or crease asymmetry on the phenylephrine test, or patients with ocular surface disease.

\section{Surgical procedures}

The operating surgeon was a new attending, who had attended several lectures, observed nearly 40 ptosis surgeries (ELA and MMCR), and performed nearly 40 upper eyelid 
blepharoplasties before direct attendance to ptosis surgeries. All surgeries were performed by a single surgeon using local anesthesia (2\% lidocaine with $1: 100,000$ epinephrine) without sedation. The amount of conjunctiva and Müller muscle excision performed was according to the phenylephrine test results, as previously recommended [3]. For MMCR, eyelid was everted by a Desmarres retractor. After the desired amount of resection was marked, eyelid was grasped with a Putterman ptosis clamp (Bausch and Lomb Storz Instruments, Manchester, MO, USA) and secured with 6-0 polypropylene suture in a continuous horizontal mattress pattern. The conjunctiva and Müller muscle were excised, and the suture was externalized and tied.

For ELA, an incision was made along the marked eyelid crease. The aponeurosis was identified and dissected from the tarsus and Müller muscle. The levator was advanced to the tarsus using a 6-0 polyglactin suture placed slightly nasal to the pupil. In approximately $10 \%$ of cases, a second or third suture was placed, but one suture was sufficient in most cases.

\section{Learning curve analysis}

Learning curves were obtained from consecutive cases using the CUSUM method. CUSUM analysis was used to quantitatively evaluate the learning curve of operation time. This method provides a graphical data of the trend in the result of consecutive surgeries since it is a plot of cumulative total of differences between separate data and the average of all data [17]. It also offers a visual representation of the learning curve $[17,20,21]$. Polynomial regression was done to provide the coefficient of determination, $\mathrm{R}^{2}$. The
CUSUM plot was obtained using QI Macros for Microsoft Excel 2016 (Microsoft Corp., Redmond, WA, USA).

\section{Clinical outcome analysis}

The clinical outcome analysis included measurement of MRD1, symmetry parameters, operative time, the intraand postoperative complications rates, and reoperation rates. MRD1 results were categorized as undercorrection (MRD1 $<3 \mathrm{~mm}$ ), optimal upper eyelid height (MRD1 $\geq 3$ and $\leq 5 \mathrm{~mm}$ ), and overcorrection (MRD1 $>5 \mathrm{~mm}$ ). Height symmetry was classified as perfect $(<0.5 \mathrm{~mm}$ difference in MRD1 between two upper eyelids), good $(\geq 0.5 \mathrm{~mm}$ and $<1$ $\mathrm{mm}$ difference in MRD1 between two upper eyelids), and fair ( $\geq 1 \mathrm{~mm}$ difference in MRD1 between two upper eyelids). Eyelid crease was classified as symmetric or asymmetric, and eyelid contour was classified as acceptable or unacceptable. Symmetry success was defined using symmetric eyelid crease, acceptable eyelid contour, and postoperative $<1 \mathrm{~mm}$ difference in MRD1 between two upper eyelids. Operative time was started after the application of local anesthesia in both techniques. Skin-to-skin operative time was defined as the time between skin incision and wound closure in ELA, while it was defined as the time between eyelid eversion using Desmarres retractor and suture externalization and ligation in MMCR [24].

\section{Statistical analysis}

IBM SPSS Statistics ver. 22.0 (IBM Corp., Armonk, NY, USA) was used for statistical analyses. KolmogorovSmirnov and Shapiro-Wilk tests were used to verify stan-

Table 1. Patient characteristics

\begin{tabular}{lccc}
\hline Characteristics & ELA $(\mathrm{n}=30)$ & MMCR $(\mathrm{n}=30)$ & $p$-value \\
\hline Age $(\mathrm{yr})$ & $53.2 \pm 9.6(36-71)$ & $40.1 \pm 9.3(25-67)$ & $<0.0001$ \\
Sex & $13(43.3)$ & $11(36.7)$ \\
$\quad$ Male & $17(56.7)$ & $19(63.3)$ & \\
Female & $1.1 \pm 0.7$ & $2.2 \pm 0.6$ & $<0.0001$ \\
Preoperative MRD1 (mm) & $3.7 \pm 0.6$ & $4.1 \pm 0.4$ & 0.019 \\
Preoperative contralateral MRD1 (mm) & $2.6 \pm 0.6$ & $1.8 \pm 0.4$ & $<0.0001$ \\
Preoperative height asymmetry (mm) & $13.9 \pm 1.9$ & $16.1 \pm 1.4$ & $<0.0001$ \\
Preoperative LF (mm) & & \\
\hline
\end{tabular}

Values are presented as mean \pm stadard deviation (range) or number (\%).

$\mathrm{ELA}=$ external levator advancement; MMCR = Müller muscle-conjunctival resection; MRD1 = margin-reflex distance; LF = levator function.

*Student's $t$-test. 
dard normal distributional assumptions. Intergroup comparisons were performed by using Student's $t$-test or Mann-Whitney $U$-test for parametric and nonparametric variables. Differences between proportions were compared using Fisher's exact test or Pearson chi-square test. A $p$ value $<0.05$ was considered statistically significant.

\section{Results}

\section{Patient characteristics}

The patient characteristics were presented in Table 1. The mean age of patients was 53.2 years in the ELA group and 40.1 years in the MMCR group. Mean preoperative MRD1 was $1.1 \pm 0.7 \mathrm{~mm}$ in the ELA group, while it was $2.2 \pm 0.6$ $\mathrm{mm}$ in the MMCR group. Significant statistical differences were observed between two groups (ELA vs. MMCR) with respect to age ( $p<0.0001$, Student's $t$-test), preoperative MRD1 in the operated and contralateral non-operated eyelid ( $p<0.0001$ and $p=0.019$, respectively, Student's $t$-test), preoperative height asymmetry ( $p<0.0001$, Student's $t$ test), and preoperative levator function ( $p<0.0001$, Student's $t$-test). Patients in the ELA group were older than the patients in the MMCR group and had lower preoperative MRD1 values.

\section{Learning curves}

Learning curves of ELA and MMCR were evaluated using CUSUM analysis based on operation times of consecutive operations (Fig. 1, 2). Cut-off values were determined

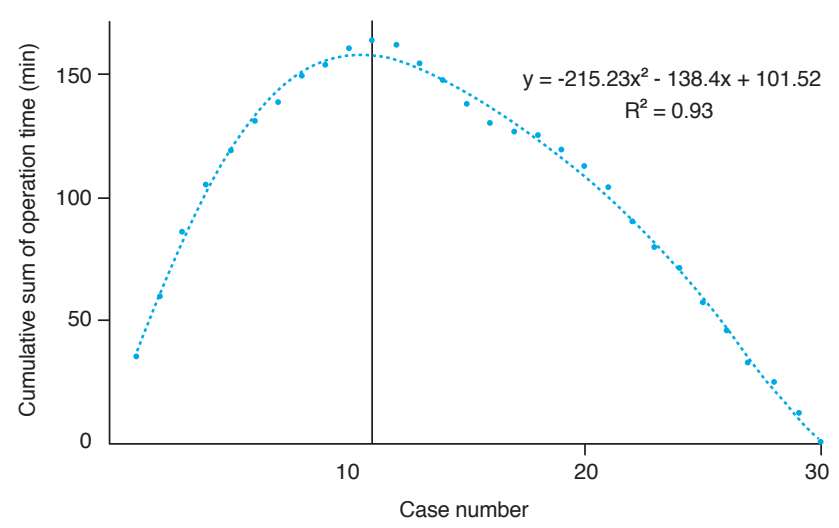

Fig. 1. Learning curve of external levator advancement using cumulative sum of operation time. according to the change in the slope of the learning curves. The cut-off cases for ELA and MMCR were found to be cases 11 and 12, respectively. CUSUM analyses revealed that the operation time stabilized after around 11 ELA surgeries and 12 MMCR surgeries. $\mathrm{R}^{2}$ value for ELA and MMCR were 0.93 and 0.91 , respectively, proving a high degree of fit. There was no significant difference in these stratified analyses. The learning curves of ELA and MMCR surgeries were found to be comparable according to CUSUM analyses.

\section{Operation time and intraoperative complications}

Mean operation time was significantly longer in ELA than MMCR ( $p=0.002$, Mann-Whitney $U$-test) (Table 2). Even though intraoperative bleeding significant enough to affect surgical manipulation was observed more in the ELA group, this difference did not reach statistical significance ( $p=0.267$, Fisher's exact test or Pearson chi-square test). Suture breaking due to extra traction during learning curve period was similar in both groups ( $p=1.0$, Fisher's exact test or Pearson chi-sqaure test). Difficulties in identifying the levator aponeurosis and in suture adjustment for lid positioning were observed exclusive to ELA surgery ( $p$ $=0.024, p<0.0001$, respectively, Fisher's exact test or Pearson chi-square test). On the other hand, exclusively in MMCR surgery, sutures were accidentally cut during the excision of Müller muscle and conjunctiva after the suturation was complete ( $p=0.02$, Fisher's exact test or Pearson chi-square test). In terms of total intraoperative complications, ELA surgery had more complications than MMCR ( $p=0.015$, Mann-Whitney $U$-test).

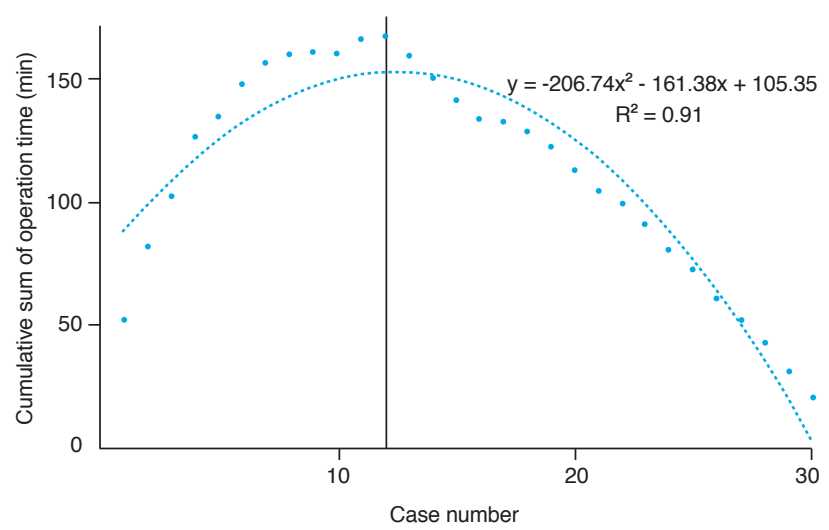

Fig. 2. Learning curve of Müller muscle-conjunctival resection using cumulative sum of operation time. 


\section{Clinical outcomes}

The clinical outcomes were presented in Table 3. Postoperative MRD1 in the operated eyelid was similar in both surgical techniques ( $p=0.754$, Student's $t$-test), while postoperative MRD1 in the contralateral non-operated eyelid was significantly lower in the ELA group $(p<0.0001$, Stu- dent's $t$-test). Postoperative height asymmetry was higher in the ELA group ( $p<0.0001$, Student's $t$-test). Symmetry success rate was significantly higher $(p<0.0001$, Fisher's exact test or Pearson chi-square test) and reoperation rate was significantly lower in the MMCR group ( $p=0.045$, Fisher's exact test or Pearson chi-square test).

Table 2. Operation time and intraoperative complications

\begin{tabular}{lccc}
\hline Characteristics & ELA $(\mathrm{n}=30)$ & MMCR $(\mathrm{n}=30)$ & $p$-value \\
\hline Operation time (min) & $45.7 \pm 13.3(32-81)$ & $34.1 \pm 14.4(21-71)$ & $0.002^{*}$ \\
Intraoperative bleeding & $12(40)$ & $7(23.3)$ & $0.267^{\dagger}$ \\
Difficulties in identifying the levator muscle & $6(20)$ & 0 & $0.024^{\dagger}$ \\
Difficulties in suture adjustment & $14(46.7)$ & $5(16.7)$ & $<0.0001^{\dagger}$ \\
Accidental cutting of the suture & 0 & $4(13.3)$ & $0.02^{\dagger}$ \\
Breaking of the suture & $5(16.7)$ & 16 & $1.0^{\dagger}$ \\
Total number of intraoperative complications & 37 & $0.015^{*}$ \\
\hline
\end{tabular}

Values are presented as mean \pm stadard deviation (range) or number (\%).

ELA $=$ external levator advancement; MMCR = Müller muscle-conjunctival resection.

${ }^{*}$ Mann-Whitney $U$-test; 'Fisher's exact test or Pearson chi-square test.

Table 3. Clinical outcomes

\begin{tabular}{|c|c|c|c|}
\hline Characteristics & $\operatorname{ELA}(n=30)$ & $\operatorname{MMCR}(n=30)$ & $p$-value \\
\hline Postoperative MRD1 (mm) & $3.7 \pm 0.9$ & $3.8 \pm 0.6$ & $0.754^{*}$ \\
\hline Postoperative contralateral MRD1 (mm) & $3.2 \pm 0.7$ & $3.9 \pm 0.5$ & $<0.0001^{*}$ \\
\hline Postoperative height asymmetry (mm) & $0.6 \pm 0.4$ & $0.2 \pm 0.3$ & $<0.0001^{*}$ \\
\hline Symmetry success & & & $<0.0001^{\dagger}$ \\
\hline Success & $13(43.3)$ & $27(90)$ & \\
\hline Unsuccess & $17(56.7)$ & $3(10)$ & \\
\hline Height asymmetry grade & & & $0.001^{\dagger}$ \\
\hline Perfect & $5(16.7)$ & $19(63.3)$ & \\
\hline Good & $14(46.7)$ & $8(26.7)$ & \\
\hline Fair & $11(36.7)$ & $3(10)$ & \\
\hline Eyelid crease & & & $0.007^{\dagger}$ \\
\hline Symmetric & $18(60)$ & $27(90)$ & \\
\hline Asymmetric & $12(40)$ & $3(10)$ & \\
\hline Eyelid contour & & & $<0.0001^{\dagger}$ \\
\hline Acceptable & $17(56.7)$ & $30(100)$ & \\
\hline Unacceptable & $13(43.3)$ & 0 & \\
\hline Undercorrection & $6(20)$ & $2(6.7)$ & $0.254^{\dagger}$ \\
\hline Optimal height correction & $22(73.3)$ & $28(93.3)$ & $0.184^{\dagger}$ \\
\hline Overcorrection & $2(6.7)$ & 0 & $0.492^{\dagger}$ \\
\hline Reoperation & $12(40)$ & $5(16.6)$ & $0.045^{\dagger}$ \\
\hline
\end{tabular}

Values are presented as mean \pm stadard deviation or number $(\%)$.

ELA = external levator advancement; $\mathrm{MMCR}=$ Müller muscle-conjunctival resection; $\mathrm{MRD} 1=$ margin-reflex distance.

"Mann-Whitney $U$-test; 'Fisher's exact test or Pearson chi-square test. 


\section{Discussion}

This study focused on evaluating the learning curves of ELA and MMCR surgeries performed by a single surgeon on patients with unilateral ptosis. Interestingly, even though the clinical outcomes of ELA were worse than MMCR, their learning curves acquired by CUSUM analysis were found to be comparable.

CUSUM is a statistical tool, which is increasingly used to objectively evaluate the learning curves of surgical techniques [11-21]. It enables us to quantitatively compare two surgical methods (in this case, ELA vs. MMCR) using statistical process control method depending on consecutive operations $[16,17]$. Recently, CUSUM analysis is commonly used to determine the learning curves, because evaluating surgical techniques based solely on clinical outcomes may decrease objectivity. One surgical technique having longer operation time than the other technique does not necessarily indicate a longer learning curve. Similarly, evaluation based on clinical outcomes and/or complication rates does not suffice either. The learning curve can also vary according to the personal practical skill of the surgeon. This can be reflected in the clinical results and surgical durations, and act as another factor that decreases objectivity. It may therefore not be sufficient to determine the learning curve just by evaluating the clinical results, complication rates, and surgical durations. Therefore, we preferred CUSUM analysis to objectively compare the learning curves of ELA and MMCR in this study.

In our study, operation time was longer $(p=0.002)$, intraoperative complication rate was higher $(p=0.015)$, postoperative success rate was lower $(p<0.0001)$ and reoperation rate was higher $(p=0.045)$ in ELA than MMCR. These clinical data may indicate that ELA is a more challenging surgery than MMCR, concluding a longer learning curve in ELA than MMCR based on only clinical data may not be accurate.

Classically, ELA is known to have a longer learning curve than MMCR. However, we found that ELA and MMCR has comparable learning curves based on CUSUM analysis. $\mathrm{R}^{2}$ values of both surgical methods proved a high degree of fit and the cut-off cases were similar. CUSUM analyses revealed that the operation time stabilized after around 11 ELA surgeries and 12 MMCR surgeries. Because our primary aim was objective learning curve analysis, we only included patients undergoing unilateral ptosis surgery and excluded patients undergoing additional surgery (blepharoplasty, brow lift, etc.) in the same session in both groups. This also helped us eliminate the confounding effects of potential complications (bleeding, suture breaking, etc.) of additional surgeries on operation time. We also aimed to make a more objective comparison by only including the period related to surgical manipulations in operation time, namely skin-to-skin operation time. Similar to our study, previous studies have reported a shorter operative time in posterior approach ptosis surgery than anterior approach [5,24].

The number of studies comparing the clinical outcomes of ELA and MMCR in the literature is very limited and the few present were published in the recent years [4-7]. Similar to these studies, we also observed more successful clinical outcomes in MMCR. However, previous studies in the literature consisted of series by experienced surgeons, while our study presents the learning curve series of a surgeon with limited experience. Therefore, it may be inaccurate to make conclusions on learning curve analysis only by comparing the clinical outcomes and operation times of both surgical techniques. However, when defining the 'learning curve,' it is also important to successfully complete the surgery. A consistent surgical duration should accompany the consistent surgical result for this definition to be met. We therefore evaluated the clinical result, surgical durations, and CUSUM analyses of the two surgical techniques together. It could also be difficult to analyze the surgical result in a small number of cases as the eyelid anatomy and function can differ in each patient.

The limitations of our study include its retrospective nature, presenting a single surgeon experience and its relatively low case number. Measurement of the MRD1 clinically may have reduced objective evaluation. The design and patient marking are also important surgical stages, and we believe there is a separate learning curve for them as well. We primarily focused on the surgical skill learning curve in this study with variable preoperative patient preparation period, and we were therefore unable to include the design and marking stages. Future studies may yield more detailed data if focused on these limitations and supported by prospective studies that co-analyses the outcomes of experienced surgeons and surgeons in training. The other limitation of this study is that the patients undergoing ELA tended to be older and more ptotic. However, the main focus of the present study was to compare the learning 
curves of ELA and MMCR. Despite these limitations, to the best of our knowledge, this is the first study to evaluate the learning curves of these two surgical techniques.

In conclusion, even though ELA procedure has longer operation time, higher intraoperative complication rate, lower postoperative success rate and higher reoperation rate than MMCR, learning curves of these two techniques acquired by CUSUM analysis were found to be comparable. Even though ELA procedure is more challenging than MMCR, comparable learning curves indicate that surgeons in training need to be encouraged to perform both techniques. The challenges and obstacles that the surgeons in training face in these two techniques may be analyzed in detail in future studies and these analyses may be used to modify and improve the training programs according to the trainees' needs.

\section{Conflict of Interest}

No potential conflict of interest relevant to this article was reported.

\section{Acknowledgements}

The authors would like to thank Ugur Ozdemir, $\mathrm{PhD}$ from University of Edinburgh for his contributions in statistical analyses.

\section{References}

1. Allen RC, Saylor MA, Nerad JA. The current state of ptosis repair: a comparison of internal and external approaches. Curr Opin Ophthalmol 2011;22:394-9.

2. Chang S, Lehrman C, Itani K, Rohrich RJ. A systematic review of comparison of upper eyelid involutional ptosis repair techniques: efficacy and complication rates. Plast Reconstr Surg 2012;129:149-57.

3. Putterman AM, Urist MJ. Muller's muscle-conjunctival resection ptosis procedure. Ophthalmic Surg 1978;9:27-32.

4. Sweeney AR, Dermarkarian CR, Williams KJ, et al. Outcomes after Muller muscle conjunctival resection versus external levator advancement in severe involutional blepharoptosis. Am J Ophthalmol 2020;217:182-8.
5. Saonanon P, Sithanon S. External levator advancement versus Muller muscle-conjunctival resection for aponeurotic blepharoptosis: a randomized clinical trial. Plast Reconstr Surg 2018;141:213e-9e.

6. Thomas GN, Chan J, Sundar G, Amrith S. Outcomes of levator advancement and Muller muscle-conjunctiva resection for the repair of upper eyelid ptosis. Orbit 2017;36:39-42.

7. Ben Simon GJ, Lee S, Schwarcz RM, et al. External levator advancement vs Muller's muscle-conjunctival resection for correction of upper eyelid involutional ptosis. $\mathrm{Am} \mathrm{J} \mathrm{Oph-}$ thalmol 2005;140:426-32.

8. Patel RM, Aakalu VK, Setabutr P, Putterman AM. Efficacy of Muller's muscle and conjunctiva resection with or without tarsectomy for the treatment of severe involutional blepharoptosis. Ophthalmic Plast Reconstr Surg 2017:33:273-8.

9. Aakalu VK, Setabutr P. Current ptosis management: a national survey of ASOPRS members. Ophthalmic Plast Reconstr Surg 2011;27:270-6.

10. Mangan MS, Atalay E, Arici C, et al. Comparison of different types of complications in the phacoemulsification surgery learning curve according to number of operations performed. Turk J Ophthalmol 2016;46:7-10.

11. Bosker R, Groen H, Hoff C, et al. Early learning effect of residents for laparoscopic sigmoid resection. J Surg Educ 2013;70:200-5.

12. Roberts HW, Wagh VB, Sung J, et al. Risk-Adjusted CUSUM analysis of the learning curve of femtosecond laser assisted cataract surgery. Curr Eye Res 2019;44:887-95.

13. Wubbels RJ, Remeijer L, Engel A, van Rooij J. The learning curve for Descemet membrane endothelial keratoplasty performed by two experienced corneal surgeons: a consecutive series of 40 cases. Acta Ophthalmol 2020;98:74-9.

14. Salowi MA, Choong YF, Goh PP, et al. CUSUM: a dynamic tool for monitoring competency in cataract surgery performance. Br J Ophthalmol 2010;94:445-9.

15. Keller J, Haynes RJ, Sparrow JM. Sequential hypothesis testing to characterise the learning curve and monitor surgical performance in retinal detachment surgery. Ophthalmologica 2016;235:157-62.

16. Li J, Xi H, Guo X, et al. Surgical outcomes and learning curve analysis of robotic gastrectomy for gastric cancer: multidimensional analysis compared with three-dimensional high-definition laparoscopic gastrectomy. Int $J$ Oncol 2019;55:733-44. 
17. Heo YJ, Kim S, Min KJ, et al. The comparison of surgical outcomes and learning curves of radical hysterectomy by laparoscopy and robotic system for cervical cancer: an experience of a single surgeon. Obstet Gynecol Sci 2018;61:46876.

18. Ramirez AG, Hu Y, Kim H, Rasmussen SK. Long-term skills retention following a randomized prospective trial on adaptive procedural training. J Surg Educ 2018;75:1589-97.

19. Biswas P, Kalbfleisch JD. A risk-adjusted CUSUM in continuous time based on the Cox model. Stat Med 2008;27:3382406.

20. Wohl H. The cusum plot: its utility in the analysis of clinical data. N Engl J Med 1977;296:1044-5.
21. Williams AK, Chalasani V, Martinez CH, et al. Cumulative summation graphs are a useful tool for monitoring positive surgical margin rates in robot-assisted radical prostatectomy. BJU Int 2011;107:1648-52.

22. Putterman AM. Margin reflex distance (MRD) 1, 2, and 3. Ophthalmic Plast Reconstr Surg 2012;28:308-11.

23. Kashkouli MB, Abdolalizadeh P, Hadi Y, et al. Change in lower eyelid position after ptosis repair in patients with unilateral myogenic versus aponeurotic blepharoptosis. Ophthalmic Plast Reconstr Surg 2019;35:383-6.

24. Sohrab MA, Lissner GS. Comparison of Fasanella-Servat and small-incision techniques for involutional ptosis repair. Ophthalmic Plast Reconstr Surg 2016;32:98-101. 